\title{
1 Rhetorical limitations in the use of frames and perspectives
}

Multiple conceptual perspectives help us understand public discourse as I have attempted to explain elsewhere in dealing with the complexity in of the problems of politics. Different conceptual frames help us address all sides of the issues in politics and help us see why policy argument is so complicated in its aims and purposes (Yoos, 2009). I introduced in that context a number of new conceptual frames and models that I found useful in guiding my own studies in rhetoric and argumentation, and I now propose to continue to add more frames and models as I transfer some of this rhetorical methodology to discussing the sciences and different forms of expertise.

Perspectives and frames have certain psychological limitations in using them if an audience is to understand readily what is being talked about. It was for this reason I kept most of my own model of methodological liberalism outlined in a sequence of simple triads (Yoos, 2009). The simplicity of such frames facilitates a reader's and listener's uptake to what is being said. As abstract, my models had the advantage of aggregating complexity and enabling us to see it logically and systematically.

Let me illustrate a mathematical and a geometric method of viewing complexity, which I shall call a mode of contrasts. This method I have already illustrated in the above mentioned published contexts (Yoos, 2009). It was best expressed I thought by a mathematical formula for measuring the numerical complexity of perspectives, oppositions, and contrasts in political frames and discussions. I used a simple formula to measure the number of loci in my model of a liberal rhetoric of practical reasons.

Contrasts are asymmetrical accounts of perspective differences. A is greater than B, and B is smaller than A. John is Mary's brother. Mary is John's sister. There are two points of view either from A or from B showing how we view these relationships as described from the two poles or perspectives. The two contrasts mark simple asymmetrical dyadic relationships and are not semantically equivalent statements. Semantically they sometimes simply logically imply each other.

My mode of enumerating contrasts then can best be illustrated by talking about the mathematical progression that allows us to calculate the numerical value of contrasts when we have a given number of contrasting terms in any frame or model. The easy way to do that is to note the mathematical progression that 'the number of contrasts equals the number of poles times the number of poles minus one.'

$$
\mathrm{C}=\mathrm{n}(\mathrm{n}-1)
$$

Contrasting perspectives from ten poles thus would then equal $10(10-1)$, which would be 90 contrasts. That is too much complexity to visualize in any form of holistic or direct attention that we might want to give to such a large complex number of 
contrasts. Too many contrasts would be an overwhelming amount of complexity in any piece of rhetoric.

As already illustrated, there are only two contrasts $2(2-1)$ for a binary perspective or a binary conceptual distinction. In a triangular set of oppositions there are two contrasts for each of the three poles A, B, and C. A contrasts with either B or with C. B contrasts with either $\mathrm{C}$ or A. C contrasts with either A or B. Three poles times two contrasts $3(3-1)$ give us six contrasts in all. The six contrasts between three terms as we will later show is a very natural way of thinking and analyzing things. We can see this pattern operate when we define things. We see it in how we think of a simple comparison or correlation of variables in talking about various factors in science, especially when we reduce or simplify our correlations to simple linear equations.

As a semantic note there are three terms contrasted in a logical definition, thus six contrasts to be made between three classes in a definition as in Figure 1.1.

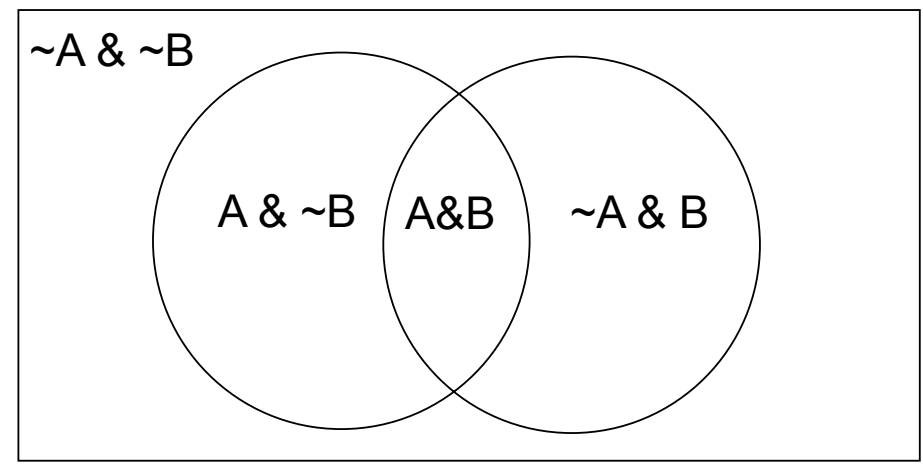

$(A \& \sim B) \&(A \& B) \&(\sim A \& \sim B) \&(\sim A \& \sim B)=1$

Figure 1.1: Venn Diagram and Conjunctive Possibilities of Combinations of two Terms and Their Complements

There is the term or the class being defined A. There is the class of which the term being defined is a subclass B. And finally there is the differentiating class $C$ that intersects with $\mathrm{B}$, the class of which the term defined is also a subclass. The division of a defining class (the class of which the term defined is a subset) by a differentiating class (another class of which the term defined is a subset) creates a class, and that class is identical or logically equivalent with the class of the term defined (thus the class of the term defined is identical with the defining class).

It is arbitrary in Figure 1.1 whether we differentiate $\mathrm{B}$ by $\mathrm{C}$ or $\mathrm{C}$ by B. It simply is a matter of perspective how one regards the defining class and the differentiating class in logical definitions. Foreground and background as in perspective in the visual arts 
are dyadic contrasts. And note that role taking in rhetoric is dyadic. The perspectives we choose to look at things depends on how we consider perspectives relevant or important. That is the principle I used in choosing the various perspectives on political argument that I presented throughout my mentioned book. Relevance and importance are key to any study of perspective that you take in looking at any policy argument, whether liberal or conservative.

The problem with essential definitions is always: 'Are they unique descriptions?', 'Are human beings (persons) uniquely rational?', 'Are human beings nothing more than persons?', 'Is a fetus a person?', 'Is a fetus a human being?'. If we are to avoid endless circling in the use of these ordinary language terms we need to avoid any specious realism and vagueness in talking about so-called natural entities as having definable essences.

Note there are many other sorts of contrasting triads especially as in the sciences comparable to those found in the logic of definition. For example in Newtonian physics, and not in Einstein's, the acceleration of a piece of mass is a function of a force applied. $\mathrm{F}=$ (f) $\mathrm{kma}$. In physics we practically apply the equation $\mathrm{F}=\mathrm{kma}$ in dynamics, where $\mathrm{k}$ is a constant depending upon the units of force, mass, and the rate of acceleration. We have thus three contrasting oppositions in the second law of dynamics between the three factors-force, mass, and acceleration - a total of six contrasts in all.

Note there is an interesting cause to the confusion that high school students have in distinguishing force and mass in physics, such as for example understanding the difference between 'a pound of force' and 'a pound of mass'. The confusion is caused by thinking that a pound of force and a pound of mass are identical units. The failure in this confusion is not noting the contrasts existing between force and mass. To understand this confusion we need to look at mass from the point of view of force and to look at force from the point of view of mass.

Importantly, and this is a point that needs emphasis, the two concepts require each other to understand the other. The two concepts are interlinked, and that is the important point that I made in discussing politics. We look at rights from the point of view of duties, and we look at duties from the point of view of rights. There is reciprocity in understanding the two concepts just as there is between mass and weight. In physics we look at force as a push or pull and then we look at mass from the point of view of a principle of inertia where we need a push or pull to overcome its reluctance to move.

Just so we need to look at rights as a privilege or a liberty that we have from a commitment or a promise (duty) that has been made. And we need to look at responsibilities as something we have created from the commitments we have made in granting people certain rights (liberties). The two concepts are interlinked in our understanding of what politics is all about.

Importantly, chemical properties are defined in chemistry by what combines with what to make new compounds. Chemical properties in this respect are chemically 
interlinked. Each element or compound is understood by their mutual interacting chemical properties. We thus look at the chemical properties of one substance from the perspective of the chemical properties of other elements and compounds, each defining the chemical properties of the other. Oxygen supports combustion, and certain chemicals are inflammable in oxygen. We understand the chemical properties of one from the chemical properties of the other.

But these simple examples from the sciences illustrating the use of triads are not truly representative of how we manage complexity in the scientific disciplines. Science deals with more complex contrasting states of affairs than those that are just to be found in simple examples of triads. The sciences have much more sophisticated tools for managing complexity not available in a rhetoric that specifically addresses non-specialist audiences. The sciences have mathematics and complex modes of modeling, using formal systems to assist in managing attention to a large numbers of factors and variables. Importantly, these models enable us to order, combine, and chunk together numerous elements to be able to deal with complexity logically in simple steps and in long sequences of logical inferences that are made one step at a time.

These methods of organization and arrangement used in the sciences require too much scrutiny and interpretation for the reading and the listening of non-specialist audiences. These formal methods are too much for audiences that are outside disciplines using formal systems and who are unable to handle complexity adequately except in simple sound bytes or aphoristic types of propositions expressed in dyads and triads.

Formal systems and mapping help the sciences reinforce powers of attention. They help those in the disciplines to remedy the psychological limits of memory, thus providing scientists with prosthetic, mnemonic visual aids in the use of graphics, script, or text, and that are importantly now being used in spreadsheets and computer printouts to engage attention to a great deal of complexity.

Such professional scientific disciplinary methods are too complicated to use in any rhetoric designed for non-specialist audiences. Scientists thus use prosthetic memory using visual texts to scrutinize large numbers of interacting variables or factors. They are able to do it by referencing and then making inferences, using a model or a text in a systematic way logically step by step. The sciences and the technologies have visual aids to memory generated by formal languages and graphics, and now they are being enhanced exponentially by computers. But to reiterate, elaborate proofs and elegant demonstrations are too complex to follow in any scientific explanation or argument that addresses non-specialist audiences unfamiliar with models such as a spread sheet.

Popular books by Albert Einstein that illustrate his theoretical physics are a lasting example of the differences between how professionals think in their disciplines and how they interpret their work for a general public. Given the limits of ordinary attention, the limits of short term memory, and the lack of a technical language that 
precise scientific terms, those who generate complex scientific argument necessarily have to resort to using a simple number of factors or variables in looking at issues in dealing with the social politics involved in the generating of the technical terms in science.

One can only deal in social political argument with the knowledge to be found in the expert disciplines by interpretations reducible to simple models or frames with simple contrasting oppositions within the constraints of usually only two or three terms. This sort of rhetorical reduction as a result of oversimplifications is often dangerous and misleading as it is prone to logical fallacies and ripe with interpretive ambiguity. We need to beware that simplifications have their own complications. 